\title{
Impacts of increasing challenge with Eimeria maxima on the growth performance and gene expression of biomarkers associated with intestinal integrity and nutrient transporters
}

\author{
Po-Yun Teng ${ }^{1}$, Janghan Choi ${ }^{1}$, Yuguo Tompkins' ${ }^{1}$, Hyun Lillehoj ${ }^{2}$ and Woo Kim ${ }^{1 *}$ (D)
}

\begin{abstract}
This study was conducted to investigate the impacts of graded severity of Eimeria maxima infection on the growth performance and intestine health of broiler chickens. Four different levels of E. maxima-challenged treatments were used, including a non-challenged control group, a low challenge (12 500 oocysts), a medium challenge (25 000 oocysts), and a high challenge dose (50 000 oocysts). There were eight replicate cages per treatment, with 12 birds in each cage, and chickens in the challenged groups orally received sporulated oocysts on day 14. Gastrointestinal permeability was measured by fluorescein isothiocyanate dextran at 5 days post-infection (dpi), whereas intestinal morphology and gene expression of nutrient transporters and tight junction proteins were determined at $6 \mathrm{dpi}$. The results demonstrate a linear reduction in growth performance, jejunal villus height, and jejunal integrity with graded challenge doses of E. maxima $(P<0.01)$. Moreover, linear regulation of nutrient transporters and tight junction proteins was a consequence of increasing Eimeria infection levels $(P<0.01)$. The linear increase of Claudin 1 , cationic amino acid transporter, glucose transporter 1, and L-type amino acid transporter genes was associated with increased severity of coccidiosis $(P<0.01)$. Furthermore, expression of nutrient transporters located at the brush border membrane were down-regulated $(P<0.01)$ with increasing E. maxima inoculation dose. In conclusion, growth performance and key intestinal integrity biomarkers in broiler chickens were adversely influenced in a dose-dependent manner by $E$. maxima infection.
\end{abstract}

Keywords: Coccidiosis, Eimeria maxima, Gastrointestinal tract health, Gastrointestinal permeability, Nutrient transporters, Tight junction proteins, Broiler chickens

\section{Introduction}

Coccidiosis, a major parasitic disease of poultry caused by several different species of the genus Eimeria, causing intestine damage, poor nutrient digestion, and diarrhea, costs approximately 14 billion United States dollars per

\footnotetext{
*Correspondence: wkkim@uga.edu

${ }^{1}$ Department of Poultry Science, University of Georgia, Athens, GA, USA

Full list of author information is available at the end of the article
}

year to the poultry industry $[1,2]$. Among seven Eimeria species found in chickens, E. maxima mainly invade the jejunum and ileum, where most dietary nutrients are digested and absorbed. Thus, E. maxima infection usually shows negative impact on growth performance because of nutrient malabsorption, and in some severe cases, high mortality in commercial farms.

Eimeria infection is known to inhibit the activities of brush border digestive enzymes such as maltase and

(c) The Author(s) 2021. This article is licensed under a Creative Commons Attribution 4.0 International License, which permits use, sharing, adaptation, distribution and reproduction in any medium or format, as long as you give appropriate credit to the original author(s) and the source, provide a link to the Creative Commons licence, and indicate if changes were made. The images or other third party material in this article are included in the article's Creative Commons licence, unless indicated otherwise in a credit line to the material. If material is not included in the article's Creative Commons licence and your intended use is not permitted by statutory regulation or exceeds the permitted use, you will need to obtain permission directly from the copyright holder. To view a copy of this licence, visit http://creativeco mmons.org/licenses/by/4.0/. The Creative Commons Public Domain Dedication waiver (http://creativecommons.org/publicdomain/ zero/1.0/) applies to the data made available in this article, unless otherwise stated in a credit line to the data. 
sucrase [2-4]. Moreover, chickens infected with $E$. maxima showed down-regulation of gene expression of nutrient transporters in the small intestine [5-7]. Nutrient transporters are protein complexes that transport dietary nutrients across the brush border membrane and the basolateral membrane of enterocytes. Glucose transporter 5 (GLUT5) is capable of transporting monosaccharides, whereas $\mathrm{Na}^{+}$-dependent amino acid transporter $\left(\mathrm{B}^{0} \mathrm{AT}\right), \mathrm{Na}^{+}$-independent amino acid transporter $\left(\mathrm{B}^{0+} \mathrm{AT}\right)$, and excitatory amino acid transporter (EAAT) can transport amino acids from the intestinal lumen to intestinal epithelial cells. Simple sugars and amino acids concentrated in the enterocytes are, however, exported into blood circulation though the nutrient transporters located at the basolateral membrane, such as glucose transporter 1 (GLUT1), glucose transporter 2 (GLUT2), cationic amino acid transporter (CAT1), L-type amino acid transporter 1 (LAT1), and $\mathrm{Na}^{+}$-dependent neutral/ cationic amino acid transporter (LAT2). A reduction in the expression of nutrient transporters and endogenous enzymes in Eimeria-infected chickens results in a decrease in the digestibility of amino acids, energy, fat, and minerals [8-13]. Given that E. maxima mainly infects the jejunum and impairs the functions of the jejunum, the main focus of this work was on evaluating E. maxima infection on biomarkers associated with intestinal integrity and nutrient transporters of broiler chickens.

Previous studies have demonstrated a strong relationship between the severity of Eimeria challenge and the growth performance of chickens [14, 15]. Additionally, digestibility of amino acids and energy was linearly decreased in a dose-dependent manner following graded Eimeria infection [11, 13]. However, one question that arises is what the potential impacts of increasing $E$. maxima infection levels on intestinal biomarkers are. Thus, the current study was conducted to investigate intestine health responses to four levels of E. maxima challenge doses. It was hypothesized that the intestinal integrity and gene expression of nutrient transporters would be linearly regulated in response to graded challenge doses of $E$. maxima. The reaction of intestinal ecosystems to different E. maxima infection severities would reveal possible mechanism of action by which parasites influence nutrient absorption and growth performance.

\section{Materials and methods}

\section{Experimental design and sample collection}

The study was conducted at the Poultry Research Center, University of Georgia, and approved by the Institutional Animal Care and Use Committee (A2018 09-006). Five hundred male broiler chickens (Cobb 500, Cobb-Vantress, Cleveland, GA, USA) were raised and fed with corn-soybean meal-based starter diet (21\% crude protein and 2,975 kcal/kg AMEn) without receiving coccidia vaccine and coccidiostats from day 0 to 14 . At day 14 , chickens were weighed individually. A total of three hundred and eighty-four birds that had similar body weight $(420 \pm 20 \mathrm{~g})$ were selected before being randomly allocated to 32 cages. Four sets of battery cages were used in the study with a floor allowance of approximately 0.049 $\mathrm{m}^{2}$ per bird. Each cage has five tiers and three cages in a row, but the top and the bottom tiers were not used in the experiment. Broiler chickens were fed with grower diet without supplementation of coccidiostats (19\% crude protein and 3,025 $\mathrm{kcal} / \mathrm{kg}$ AMEn) from day 14 to day 20. Eight replicate cages were allotted for each treatment group, with 12 chickens per cage. Feed and water were provided ad libitum and experimental animals were raised in the same room where the environmental temperature was set at $27{ }^{\circ} \mathrm{C}$ on day 14 and reduced to $24{ }^{\circ} \mathrm{C}$ by decreasing $1^{\circ}$ every other day according to the recommendation of the Cobb Broiler Management Guide [16].

There were four levels of E. maxima-challenged treatment groups: a low challenge dose, a medium challenge dose and a high challenge dose, and a non-challenged control group (Control). At the beginning of the study, the chickens in the low-dose treatment (Low) group were gavaged with 12500 sporulated oocysts of E. maxima per bird; the chickens in the medium-dose treatment (Medium) were gavaged with 25000 sporulated oocysts per bird; the chickens in the high-dose treatment (High) were gavaged with 50000 sporulated oocysts per bird; whereas, the non-challenged chickens were gavaged with $1 \mathrm{~mL}$ of water. The dosage levels for the current study were chosen based on previous studies [11,13].

The E. maxima used in the study were isolated and propagated from a North Carolina field strain. In brief, a single oocyst was obtained using a pipette under a light microscope. The chicken that was used for oocyst propagation was raised in a disinfected isolator and infected with one oocyst at the age of 14 days. At $7 \mathrm{dpi}$, feces were collected from the isolator. The oocysts were separated from feces by salt flotation and sporulated with an air pump at room temperature. Three rounds of passages were performed to gain sufficient E. maxima oocysts for the current experiment.

The feed intake (FI) and body weight (BW) of chickens per pen were recorded on 0 and 6-dpi to calculate body weight gain (BWG) and feed conversion rate (FCR) during the experimental period. Mortalities were recorded to adjust FI and FCR when chickens died before the trial termination. On $5 \mathrm{dpi}$, one chicken per cage was sacrificed by cervical dislocation to measure gastrointestinal permeability. On $6 \mathrm{dpi}$, three to five centimeter long sections of mid-jejunum were collected from one bird per 
cage. Intestinal digesta was gently flushed out of the tissue using phosphate buffer saline. Afterward, the intestinal tissue was immediately fixed in $10 \%$ formalin to determine intestinal morphology. For gene expression analysis, another five centimeters long section of midjejunum was collected. The jejunal mucosa was gently scraped with a blunt microslide, immediately snap-frozen in the liquid nitrogen, and stored at $-80{ }^{\circ} \mathrm{C}$ until further processing.

\section{Gastrointestinal permeability}

One chicken per cage was gavaged with $1 \mathrm{~mL}$ of fluorescein isothiocyanate dextran (FITC-d; $2.2 \mathrm{mg} / \mathrm{mL}, \mathrm{MW}$ 4000; Sigma-Aldrich, Canada) to measure gastrointestinal permeability $[13,17]$. At $2 \mathrm{~h}$ post-inoculation, blood was collected from chickens after euthanasia by cervical dislocation. Blood samples were stored in a dark container for an additional $2 \mathrm{~h}$ at room temperature before centrifugation at $1000 \times g$ for $15 \mathrm{~min}$. A hundred microliter of serum was transferred from the blood sampling tubes to a dark 96-well microplate. Five levels of FITC-d standard solution were formulated with the stock solution $(2.2 \mathrm{mg} / \mathrm{mL})$ and the pool of serum from 10 additional unchallenged chickens raised in the same house. The standard solution and test samples were measured at an excitation wavelength of $485 \mathrm{~nm}$ and an emission wavelength of $528 \mathrm{~nm}$ with a microplate reader (Spectramax M5, Molecular Devices, San Jose, CA, USA). The concentration of FITC-d in the serum was calculated using a standard curve. The processing of blood and preparation of standard solution were performed under a dark environment in order to protect FITC-d from light exposure.

\section{Intestinal morphology}

The fixed intestinal tissues were removed from the $10 \%$ formalin and embedded in paraffin. Afterward, $4 \mu \mathrm{m}$ tissue slides were made from the embedded samples and stained with hematoxylin and eosin. The intestinal morphology of each slide was observed and captured by a light microscope with $2 \times$ magnification (BZ-X800, Keyence Inc, Itasca, IL, USA). Villus height and crypt depth was measured by Image J (Image Processing and Analysis in Java-ImageJ 1.50i, National Institutes of Health) with five representative villi and crypts per slide. Additionally, the ratio of villi height to crypt depth was calculated by the average value of villi height and crypt depth from each slide.

\section{Real-time PCR analysis}

Total RNA from each sample was extracted and homogenized in QIAzol Lysis Reagents (Qiagen, Germantown, MD, USA) with a bead beater. The RNA quantity was determined by a NanoDrop 2000 spectrophotometer (Thermo Fisher Scientific, MA, USA). The extracted RNA was diluted to the same concentration for each sample and reverse-transcribed to cDNA by the High Capacity cDNA synthesis kits (Applied BioSystems, Life Technologies, CA, USA). In the real-time PCR reactions, cDNA samples were mixed with SYBR Green Master mix (Bio-Rad Laboratories, Hercules, CA, USA) for analysis in the Step One thermo-cycler (Applied Biosystem, Foster City, CA, USA) in duplicates. The $2^{-\Delta \Delta \mathrm{Ct}}$ method was used to analyze target gene expression compared to a housekeeping gene [18]. GAPDH was selected to be the reference gene based on the most stable $\mathrm{Ct}$ value among other housekeeping genes that has been tested (beta-actin, 18S, and hydroxymethylbilane synthase). The variation of $\mathrm{Ct}$ value was within one Ct-cycle among all treatments and replicates. Additionally, there was no linear effect observed in the Ct value of the reference gene in response to the graded levels of Eimeria challenge doses. The mean $\Delta \mathrm{Ct}$ from the non-challenged control was used as the base standard to adjust the fold changes of other treatments. The integral proteins and membrane associated guanylate kinase homologue proteins ( $\mathrm{ZO}$ family) at the tight junction complex were evaluated in the study, including occludin (OCLDN), Zonula occludens 1 (ZO1), Zonula occludens 2 (ZO2), junctional adhesion molecule 2 (JAM2), claudin 1 (CLDN1), and claudin 2 (CLDN2). Moreover, gene expression of mucin (mucin 2, MUC2), monosaccharide transporters (GLUT1, GLUT2, GLUT5, and, SGLT1), and amino acids transporters $\left(\mathrm{B}^{0} \mathrm{AT}, \mathrm{B}^{0+} \mathrm{AT}\right.$, EAAT, CAT1, LAT1, and LAT2) were determined in the mucosa of the jejunum. The sequences of the forward and reverse primers are listed in Table 1.

\section{Statistical analyses}

All data were analyzed in the PROC GLM program of SAS software (SAS Institute Inc., Cary, NC, USA). For bird mortality, the number of dead birds per pen was transformed by the formula below [19].

$$
\text { Transformed data }=\sqrt{\text { Dead birds }+1}
$$

Orthogonal polynomial contrasts were used to evaluate the linear and quadratic impacts of graded E. maxima challenge doses on growth performance, mortality, and parameters of intestine health. The CONTRAST statements within PROC GLM were used to perform the analyses and the statistical significance was set at $P<0.05$ [20].

\section{Results}

\section{Growth performance and mortality}

Increasing the inoculation doses of E. maxima linearly and quadratically decreased the BW, BWG, and FI of birds $(P<0.0001$, Table 2$)$. Additionally, the graded 
Table 1 List of primers used for qPCR

\begin{tabular}{|c|c|c|c|}
\hline Gene symbol & Accession Number & Forward primer & Reverse primer \\
\hline $\mathrm{GAPDH}^{\mathrm{a}}$ & NM_204305.1 & ССTCTCTGGCAAAGTCCAAG & GGTCACGCTCCTGGAAGATA \\
\hline CLDN1 $1^{\mathrm{b}}$ & NM_001013611.2 & TGGAGGATGACCAGGTGAAGA & CGAGCCACTCTGTTGCCATA \\
\hline$C L D N 2^{b}$ & NM_001277622.1 & CCTGCTCACCCTCATTGGAG & GCTGAACTCACTCTTGGGCT \\
\hline OCLDN ${ }^{b}$ & NM_205128.1 & ACGGCAGCACCTACCTCAA & GGCGAAGAAGCAGATGAG \\
\hline $\mathrm{ZO} 1^{\mathrm{b}}$ & XM_015278981.2 & CAACTGGTGTGGGTTTCTGAA & TCACTACCAGGAGCTGAGAGGTAA \\
\hline $\mathrm{ZO} 2^{\mathrm{b}}$ & NM_204918.1 & ATCCAAGAAGGCACCTCAGC & CATCCTCCCGAACAATGC \\
\hline $\mathrm{JAM} 2^{\mathrm{b}}$ & NM_001006257.1 & AGCCTCAAATGGGATTGGATT & CATCAACTTGCATTCGCTTCA \\
\hline MUC2 ${ }^{C}$ & JX284122.1 & ATGCGATGTTAACACAGGACTC & GTGGAGCACAGCAGACTTTG \\
\hline GLUT5 (SLC2A5) ${ }^{d}$ & XM_417596.6 & TTGCTGGCTTTGGGTTGTG & GGAGGTTGAGGGCCAAAGTC \\
\hline SGLT1 (SLC5A1) d & NM_001293240.1 & GCCGTGGCCAGGGCTTA & CAATAACCTGATCTGTGCACCAGT \\
\hline$B^{0} A T(S L C 6 A 19)^{d}$ & XM_419056.6 & GGGTTTTGTGTTGGCTTAGGAA & TCCATGGCTCTGGCAGAGAT \\
\hline$B^{0+} A T($ SLC7A9) & NM_001199133.1 & CAGTAGTGAATTCTCTGAGTGTGAAGCT & GCAATGATTGCCACAACTACCA \\
\hline EAAT $(S L C 1 A 1)^{d}$ & XM_424930.6 & TGCTGCTTTGGATTCCAGTGT & AGCAATGACTGTAGTGCAGAAGTAATATATG \\
\hline GLUT2 (SLC2A2) $)^{d}$ & NM_207178.1 & TCATTGTAGCTGAGCTGTT & CGAAGACAACGAACACATAC \\
\hline CAT1 (SLC7A1)d & XM_015277945.2 & CCAAGCACGCTGATAAAG & TACTCACAATAGGAAGAAGGG \\
\hline LAT2 (SLC7A6) ${ }^{d}$ & XM_025154295.1 & TCAGCTTCAGTTACTGGTT & GCACAACCACGAGAAATAC \\
\hline GLUT1(SLC2A1) d & NM_205209.1 & CTTTGTCAACCGCTTTGG & TGTGCCCCGGAGCTTCT \\
\hline LAT1 (SLC7A5) & NM_001030579.2 & GATTGCAACGGGTGATGTGA & CCCCACACCCACTTTTGTTT \\
\hline
\end{tabular}

GAPDH glyceraldehyde-3-phosphate dehydrogenase, CLDN1 Claudin 1 [49], CLDN2 Claudin 2 [50], OCLDN Occludin [51], ZO1 Zonula occludens 1 [52], ZO2 Zonula occludens 2 [53], JAM2 Junctional adhesion molecule 2 [48], MUC2 Mucin [47], GLUT5 Glucose transporter 5, SGLT1 sodium glucose transporter 1, B ${ }^{0}$ AT Na ${ }^{+}$dependent amino acid transporter, $\mathrm{B}^{0+} \mathrm{AT} \mathrm{Na}{ }^{+}$-independent amino acid transporter, EAAT Excitatory amino acid transporter, GLUT2 Glucose transporter 2, CAT1 Cationic amino acid transporter, LAT2 $\mathrm{Na}^{+}$-dependent neutral/cationic amino acid transporter, GLUT1 Glucose transporter 1, LAT1 L-type amino acid transporter 1.

${ }^{\text {a }}$ Housekeeping gene.

${ }^{\mathrm{b}}$ Tight junction proteins.

cMucin.

${ }^{\mathrm{d}}$ Nutrient transporters [24].

challenge levels resulted in linear increases in FCR from 0 to $6 \mathrm{dpi}(P<0.0001)$. The FCR for the High group increased from 1.40 to 3.14 compared to the Control group. The chickens challenged with E. maxima had a $62 \%$ reduction in BWG compared to the Control group. Moreover, the mortality of birds was linearly increased in a dose-dependent manner $(P<0.0001)$.

\section{Intestinal morphology}

Linear reductions for villus height in the jejunum were observed with increasing challenge oocyst doses $(P<0.0001$; Table 3 , Figure 1$)$. In addition, the crypt depth of the jejunum also exhibited a linear response to the graded E. maxima infection $(P<0.05)$, whereas the ratio of villus height to crypt depth in the jejunum

Table 2 Effects of graded E. maxima infection on growth performance and mortality of broiler chickens from 0 to 6-dpi

\begin{tabular}{|c|c|c|c|c|c|c|}
\hline Items $^{a}$ & Control & Low & Medium & High & Linear $^{b} P$ value & Quadratic ${ }^{\mathrm{b}} P$ value \\
\hline BW (g) & $832 \pm 8^{c}$ & $637 \pm 5$ & $565 \pm 10$ & $524 \pm 6$ & $<0.0001$ & $<0.0001$ \\
\hline BWG (g) & $415 \pm 8$ & $203 \pm 5$ & $139 \pm 9$ & $132 \pm 6$ & $<0.0001$ & $<0.0001$ \\
\hline $\mathrm{Fl}(\mathrm{g})$ & $579 \pm 9$ & $435 \pm 17$ & $414 \pm 17$ & $410 \pm 16$ & $<0.0001$ & $<0.0001$ \\
\hline FCR & $1.40 \pm 0.02$ & $2.15 \pm 0.07$ & $3.04 \pm 0.15$ & $3.14 \pm 0.12$ & $<0.0001$ & $<0.0001$ \\
\hline Mortality (\%) & $1.04 \pm 1.04$ & $4.17 \pm 1.57$ & $19.79 \pm 1.04$ & $23.96 \pm 1.04$ & $<0.0001$ & 0.0713 \\
\hline
\end{tabular}

The study evaluated the effects of graded challenge of E. maxima on growth performance and gastrointestinal biomarkers in the broiler chickens. Birds were challenged with E. maxima on day 14.

a Low, 12500 oocysts of E. maxima; Medium, 25000 oocysts of E. maxima; High, 50000 oocysts of E. maxima; BW body weight, BWG body weight gain, FI feed intake, FCR feed conversion rate.

${ }^{\mathrm{b}}$ The $P$ values indicate whether the analyzed parameters linearly or quadratically responded to the graded challenge doses of $E$. maxima.

${ }^{c}$ Mean \pm SD. 
Table 3 Effects of graded E. maxima infection on intestinal morphology of broiler chickens on 6-dpi

\begin{tabular}{|c|c|c|c|c|c|c|}
\hline Items $^{a}$ & Control & Low & Medium & High & Linear $^{b} P$ value & $\begin{array}{l}\text { Quadratic } \\
P \text { value }\end{array}$ \\
\hline \multicolumn{7}{|l|}{ Jejunum ( $\mu \mathrm{m})$} \\
\hline Villi height & $1155 \pm 46^{d}$ & $911 \pm 27$ & $900 \pm 31$ & $800 \pm 22$ & $<0.0001$ & 0.006 \\
\hline Crypt depth & $205 \pm 9$ & $216 \pm 34$ & $182 \pm 6$ & $171 \pm 7$ & 0.002 & 0.804 \\
\hline Ratio $^{c}$ & $5.85 \pm 0.35$ & $4.48 \pm 0.26$ & $5.15 \pm 0.22$ & $4.96 \pm 0.20$ & 0.143 & 0.060 \\
\hline
\end{tabular}

The study evaluated the effects of graded challenge of E. maxima on growth performance and gastrointestinal biomarkers in the broiler chickens. Birds were challenged with E. maxima on day 14. Intestinal tissue was collected on 6-dpi.

a Low, 12500 oocysts of E. maxima; Medium, 25000 oocysts of E. maxima; High, 50000 oocysts of E. maxima.

${ }^{\mathrm{b}}$ The $P$ values indicate whether the analyzed parameters linearly or quadratically responded to the graded challenge doses of $E$. maxima.

c Ratio of villi height to crypt depth

${ }^{\mathrm{d}}$ Mean $\pm S D$.

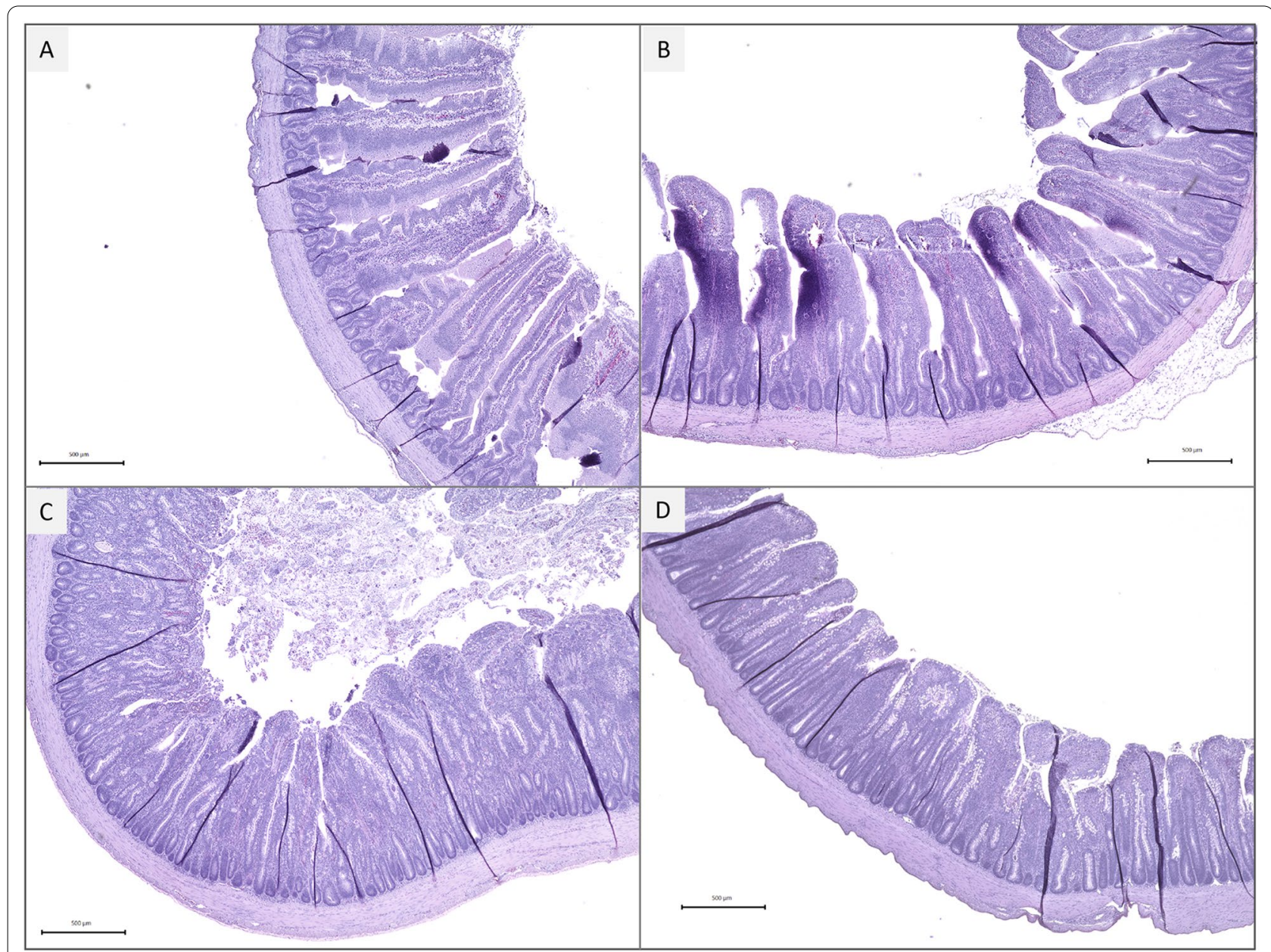

Figure 1 Effects of graded E. maxima infection on intestinal morphology (jejunum) of broiler chickens at 6-dpi. A Control; B Low; C Medium; $\mathbf{D}$ High. The study evaluated the effects of graded challenge of E. maxima on growth performance and gastrointestinal biomarkers in the broiler chicken. Birds were challenged with E. maxima on day 14. Low, 12500 oocysts of E. maxima; Medium, 25000 oocysts of E. maxima; High, 50000 oocysts of E. maxima. 
showed a trend of a quadratic effect $(P<0.1)$. Furthermore, the chickens challenged with Low or High doses of E. maxima exhibited numerically lower ratio of villi height to crypt depth in the jejunum compared to the Control group.

\section{Gastrointestinal permeability and gene expression of tight junction proteins}

The results of gastrointestinal permeability at $5 \mathrm{dpi}$ ) (Figure 2) indicated that increasing the severity of E. maxima infection linearly elevated the FITC-d levels in the serum $(P<0.01)$, suggesting that gastrointestinal leakage was linearly increased in response to higher levels of $E$. maxima challenge. Furthermore, linear responses were observed for the gene expression of several tight junction proteins and the mucin at $6 \mathrm{dpi}$ ) (Figure 3). The mRNA levels of OCLDN, ZO1, CLDN2, and MUC2 were linearly and quadratically decreased, whereas the gene expression of CLDN1 was significantly upregulated when birds were challenged with higher levels of oocysts $(P<0.01)$. In addition, there was a quadratic effect on the gene expression of JAM2 in the mucosa of the jejunum $(P<0.05)$. JAM2 was upregulated in the Medium group at levels two times higher than that in the Control group.
However, there was no significant difference in gene expression of $\mathrm{ZO} 2$ among all treatments.

\section{Gene expression of nutrient transporters}

Gene expression of nutrient transporters located at the brush border of the mucosa was linearly and quadratically reduced in response to increasing doses of Eimeria challenge $(P<0.01)$ (Figure 4$)$. The challenged chickens exhibited reduced gene expression for GLUT5 (Low, 90\% of expression reduction compared to the non-challenged group; Medium, 92\%; High, 91\%), SGLT1(Low, 75\% of expression reduction compared to the non-challenged group; Medium, $80 \%$; High, $76 \%$ ), $\mathrm{B}^{0} \mathrm{AT}$ (Low, $57 \%$ of expression reduction compared to the non-challenged group; Medium, 73\%; High, 66\%), $\mathrm{B}^{0+} \mathrm{AT}$ (Low, $86 \%$ of expression reduction compared to the non-challenged group; Medium, 92\%; High, 93\%), and EAAT (Low, 74\% of expression reduction compared to the non-challenged group; Medium, 82\%; High, 82\%) compared to the Control group. Moreover, linear and quadratic reductions in GLUT2 and LAT2 was observed in the basolateral membrane of mucosa in response to the graded increasing level of E. maxima infection $(P<0.01)$. Gene expression of GLUT2 and LAT2 in challenged chickens was

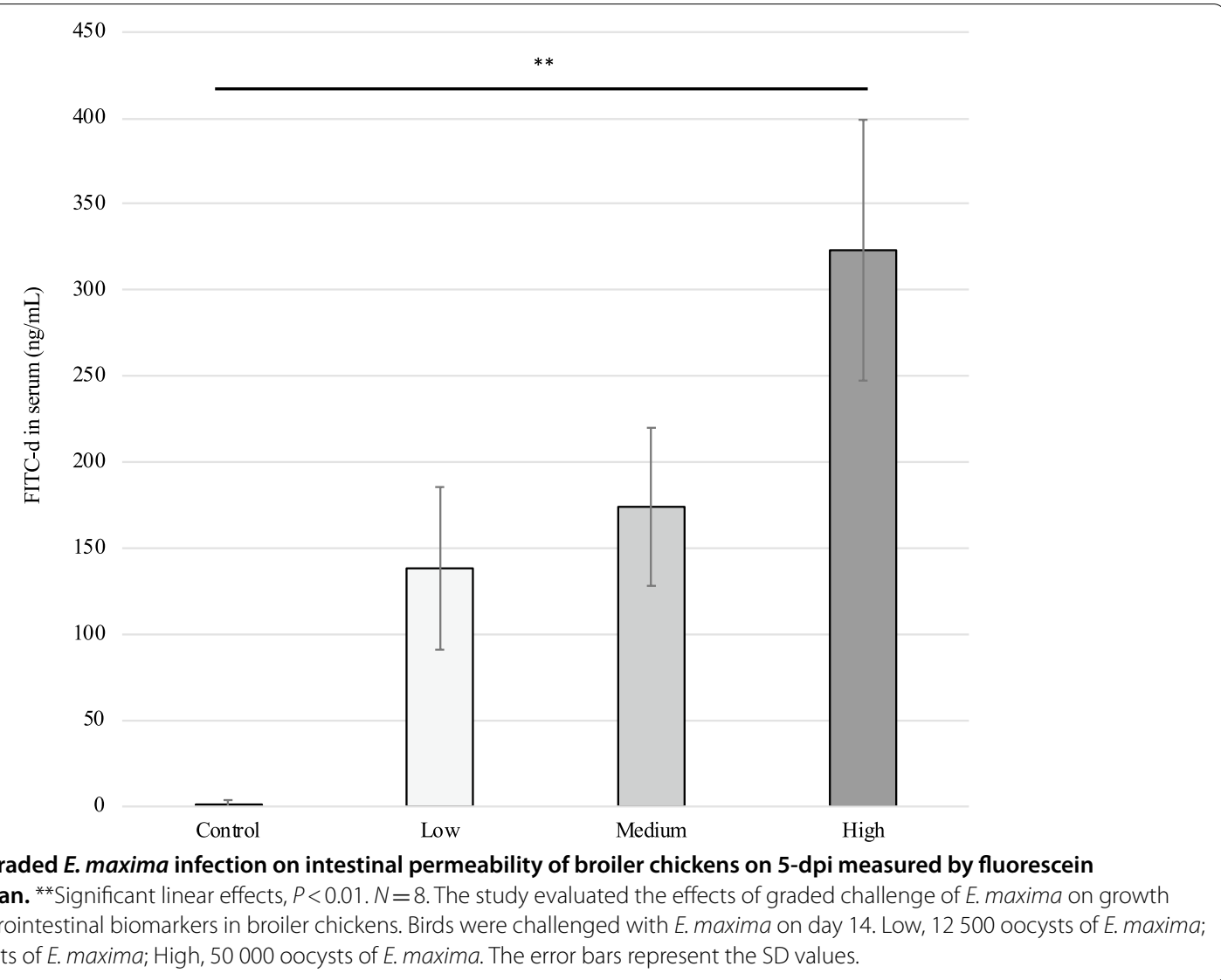




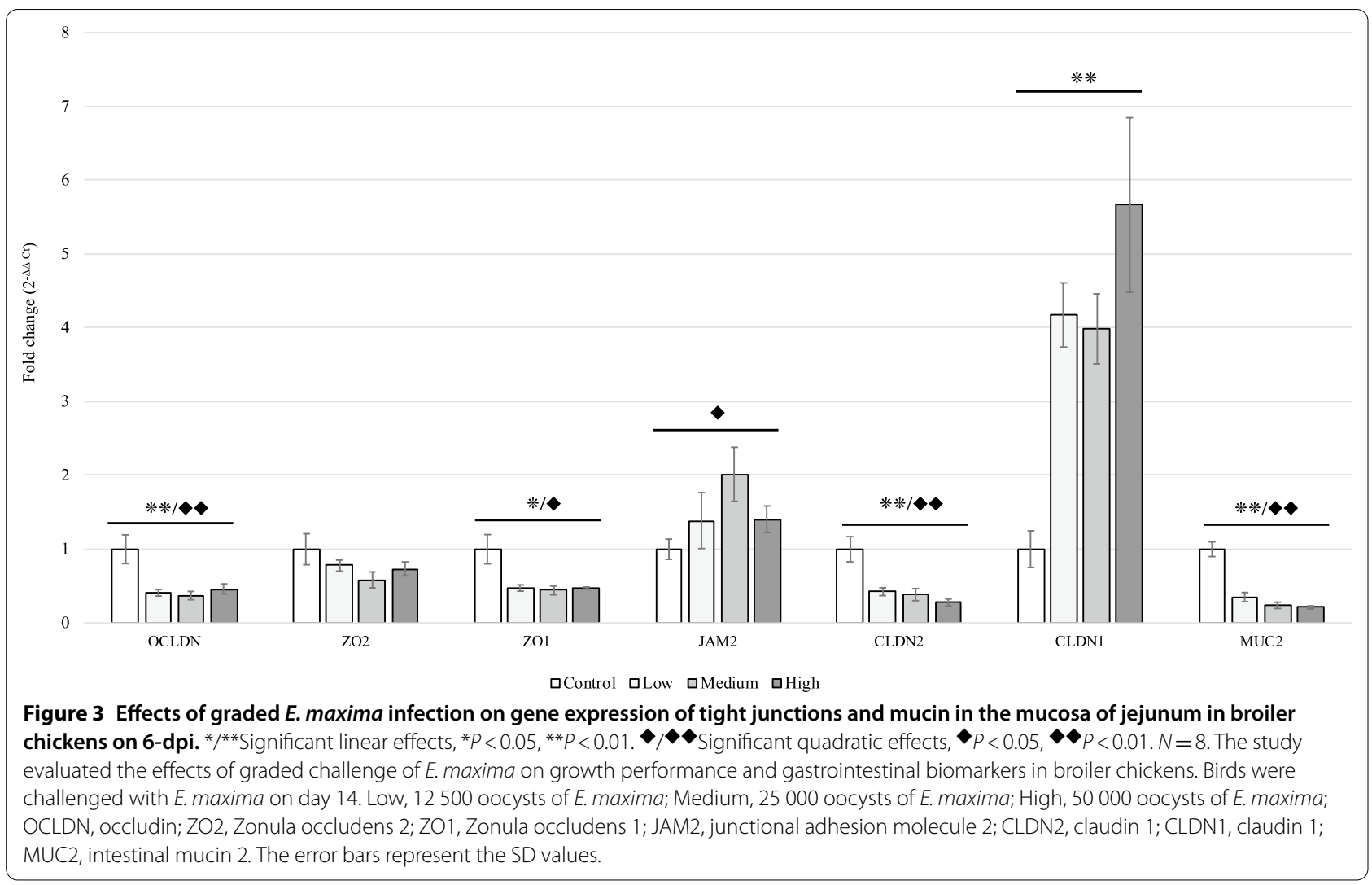

downregulated to less than $20 \%$ of the Control group. CAT1, GLUT1, and LAT1, located at the basolateral membrane, were, however, linearly and quadratically upregulated with increasing inoculation doses of $E$. maxima $(P<0.05$, Figure 5$)$. The gene expression of LAT1 in the Medium group was 21 times higher than that in the Control group, whereas those of CAT1 and GLUT1 were increased to $207 \%$ and $328 \%$, respectively, in the Control group (Figure 5).

\section{Discussion}

Linear reduction in growth performance in response to the increase in inoculation doses of E. maxima could be attributed to several factors. First, FI plays a crucial role in maintaining the intestinal health status and BWG of chickens. In the present study, FI was significantly decreased in the challenged chickens because of sickness and lethargy after the E. maxima infection. The linear decrease in feed intake was strongly associated with BW when chickens were challenged with graded levels of mixed Eimeria spp. [13]. It was reported that the correlation coefficient between BW and FI was up to 0.8 in our previous study [13]. Moreover, a meta-analysis indicated that reduced FI resulted in approximately $90 \%$ of the loss of total BWG in chickens infected by Eimeria spp. [21]. Apart from E. maxima infection, increasing challenge levels of $E$. acervulina, E. tenella, or mixed Eimeria species all caused linear inhibition of the growth performance of chickens $[11,14]$. However, among seven species, E. maxima had the most significant influence on BWG because of its preferable infection sites at the jejunum and ileum, where most dietary nutrients are supposed to be digested and absorbed [21].

The reduced nutrient digestibility could be another factor that contributes to the poor growth performance in chickens challenged with coccidia. It has been reported that Eimeria infection inhibits the apparent ileal digestibility of amino acids, energy, and minerals [11-13]. The decrease in nutrient digestibility in coccidiosis might be associated with the expression of nutrient transporters in the jejunum. The present results demonstrate that the increasing severity of E. maxima infection linearly reduced the expression of nutrient transporters located at the brush border of epithelial cells. The downregulation of GLUT5 and SGLT1 could decrease fructose, glucose, and galactose absorption [22]. Additionally, the increase in E. maxima challenge linearly reduced the gene expression of $\mathrm{B}^{0} \mathrm{AT}, \mathrm{B}^{0+} \mathrm{AT}$, and EAAT. $\mathrm{B}^{0} \mathrm{AT}$ is a nutrient transporter that takes up a broad range of neutral amino acids from the intestinal lumen to epithelial 

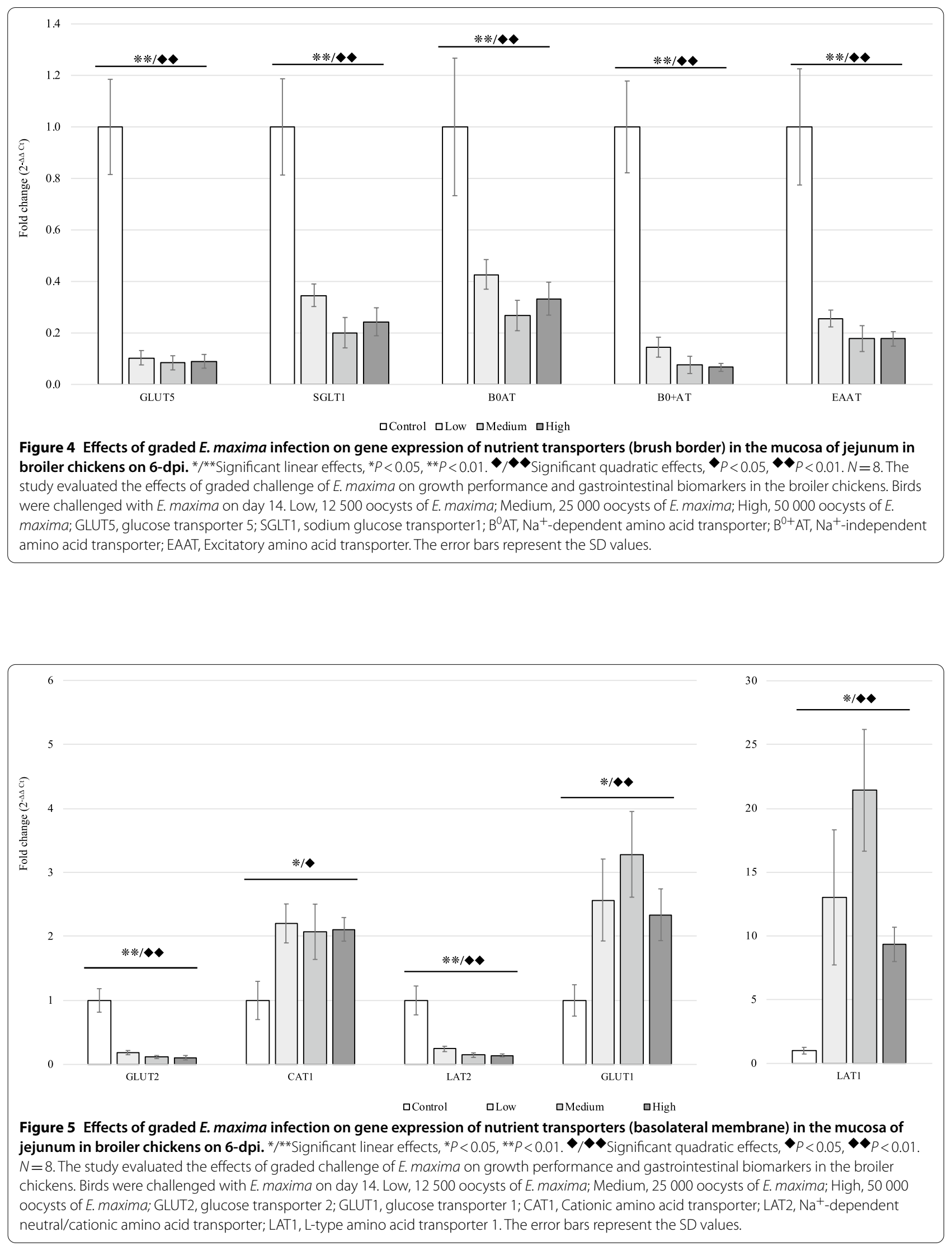
cells; thus, a reduced expression of $\mathrm{B}^{0} \mathrm{AT}$ decreases the transport of neutral amino acids [23, 24]. Moreover, the reduced levels of neutral amino acids further influence the uptake of cationic amino acids because neutral amino acids accumulate in enterocytes to exchange cationic amino acids by the amino acid exchanger complex composed of $\mathrm{B}^{0+} \mathrm{AT}$ and rBAT [23]. The downregulation of $\mathrm{B}^{0+} \mathrm{AT}$ in the current study indicates a depressed uptake of essential cationic amino acids, such as Arg and Lys, in response to E. maxima infection. These amino acids are important for the growth of the host and the development of immunity and antioxidative responses; therefore, the decreased availability of cationic amino acids might further impact host health status, such as immune responses against pathogen invasion and the growth performance of chickens [25-27]. Gene expression of EAAT, a sodium-dependent nutrient transporter for anionic amino acids including glutamate and aspartic acid [28], was also linearly reduced in response to the increase in challenge doses in the present study. It is speculated that the downregulation of EAAT reduced the uptake of glutamate which would accelerate cell death and inhibit parasite replication [6] because glutamate is the main source of energy metabolism in enterocytes. In the present study, although the expression of nutrient transporters located at the brush border was linearly reduced with increasing levels of challenge, it should be noted that the chickens in the Low group decreased the expression of the target genes to less than $50 \%$ of the Control group. The results suggest that coccidiosis causes significant impacts on the gene expression of nutrient transporters regardless of the severity of the infection.

Similar to the expression of nutrient transporters located at the brush border of the enterocytes, GLUT2, CAT1, LAT1, GLUT1, and LAT2 at the basolateral membrane were linearly regulated by increasing $E$. maxima infection in the current study. The gene expression of GLUT2 and LAT2 was downregulated, but CAT1, GLUT1, and LAT1 were upregulated by coccidiosis. The current results were in agreement with a previous study that reported increased expression of GLUT1, LAT1, and CAT1 in the jejunum after chickens were challenged with E. maxima [6]. Furthermore, it was reported that the expression of LAT2 and GLUT2 is reduced in the jejunum of laying hens by coccidia infection [5]. GLUT1 and GLUT2 are capable of transporting hydrolyzed carbohydrate molecules from enterocytes to the blood circulation, whereas LAT1, LAT2, and CAT transport amino acids. The mechanism of action by which coccidiosis increased GLUT1, LAT1, and CAT1, but decreased GLUT2 and LAT2 remains, however, unknown.

In the current study, the gene expression of LAT1 was increased 13-, 21-, and ninefold in the Low, Medium, and High groups, respectively. Similarly, it was reported that E. maxima infection causes a 19-fold change in LAT1 compared to that in non-challenged chickens [7]. LAT1 transports neutral, branched, and aromatic amino acids, such as Met and Leu. The upregulated expression of LAT1 enhances the efflux of neutral amino acids from epithelial cells. Moreover, the expression of CAT1, which transports cationic amino acids such as Cys, Arg, and Lys, was linearly increased in response to graded $E$. maxima challenge. The current findings were similar to those of studies indicating the upregulation of CAT1 and LAT1 after Eimeria infection [24, 29]. Both studies suggested that coccidiosis may deplete essential amino acids in infected cells by upregulating amino acid transporters at the basolateral membrane and downregulating nutrient transporters at the brush border membrane. Nutrient depletion, such as glutamine deficiency, may trigger spontaneous apoptosis in intestinal epithelial cell lines [30, 31]. The "apoptosis-induced proliferation" is crucial for tissue regeneration and recovery [32]. It has been reported that coccidiosis could accelerate the renewal of intestinal epithelial cells [33, 34]. Moreover, the increased epithelial cell turnover rate may expel parasites from the host's intestine [35]; therefore, regulating gene expression in the enterocytes may lead to nutrient depletion and cell apoptosis which remove parasites from the host with the assistance of an increased proliferation of epithelial cells. Furthermore, $\mathrm{Su}$ et al. [5] have proposed two possible mechanisms that would cause the alternations of nutrient transporters during coccidiosis. They speculated that Eimeria infection could directly influence gene expression of epithelial cells; otherwise, the regulations could be cell-mediated consequences in response to parasite invasion. None of the hypotheses has been proven, but the latter possibility is favored by the authors.

Even though significant linear effects were observed for CAT1 and LAT1, the Medium group had numerically greater gene expression than the High group in the present study. These findings might be attributed to the crowding effect when there was no available room in the intestine of the host for parasites to develop their life cycles [36]. The numbers of parasites in the high challenge treatment may exceed the threshold of the host's capability that could be utilized for Eimeria's replication. The crowding effect might not only influence the expression of CAT1 and LAT1 in the High challenge treatment, but also the SGLT1, BOAT, and EAAT, as these target genes did not perform exact linear trends according to the increasing infection severity. Our previous study also observed that the crowding effect would influence intestinal permeability. Severe Eimeria infection would lead to an early peak of gastrointestinal leakage at $5 \mathrm{dpi}$, instead of $6 \mathrm{dpi}$, whereas mild infection reached the peak a day 
later [13]. The regulation of nutrient transporters by a crowding effect may further influence nutrient digestibility of chickens. The birds that received a medium inoculation dose of Eimeria had lower digestibility of amino acids and energy than those challenged with a relative higher dose $[11,13]$. Because the intestinal ecosystems changed dramatically during Eimeria infection, further research might be needed to determine the daily expression of nutrient transporters and nutrient digestibility to understand the dynamic change of intestine status in response to different Eimeria infection levels. Although the threshold of parasite development still remains unclear, the current study could conclude that the transportation of amino acids and carbohydrates across the brush border and basolateral membrane was linearly responding to the graded challenge doses of E. maxima, but the linear trend might be influenced by a crowding effect, especially at the high challenge dose.

Coccidiosis has shown significant impacts on the intestinal integrity of chickens. The graded oocyst inoculation caused cell destruction in a dose-dependent manner, consequently influencing the gene expression of tight junction proteins and gastrointestinal permeability. FITC-d was used to determine the intestinal integrity of chickens in the current study. The greater amounts of FITC-d in the serum indicates more severe intestine damage caused by Eimeria infection. Our findings suggest that gastrointestinal permeability was linearly enhanced in response to graded E. maxima challenge. The current results agreed with previous studies that reported the linear regulation of gastrointestinal permeability with increasing Eimeria infection $[11,13]$.

Coccidiosis influences transcellular translocation by impairing intestinal epithelial cells as well as paracellular translocation by breaking the tight junctions between enterocytes. Tight junction proteins construct a complex structure connecting cells to impede the transportation of large molecules across the intestinal border. This complex is composed of several types of proteins, such as CLDN, OCLDN, JAM, and ZO families [37]. The current findings show that the expression of all tight junction proteins except $\mathrm{ZO} 2$ was significantly changed during coccidiosis. CLDN1 was linearly and JAM2 was quadratically increased at $6 \mathrm{dpi}$ in response to graded E. maxima challenge. The CLDN and JAM families are responsible for tight junction strand formation and membrane apposition, respectively [38]. Moreover, both CLDN and JAM families coordinately regulate tight junction complex formation and paracellular translocation [38]. The activation of CLDN1 might be associated with the increase in cytokines caused by parasitic infection. TNF-alpha and IFN-gamma have been concluded to be the main cytokines produced by the host against Eimeria spp. $[39,40]$. It was reported that TNF-alpha and IFNgamma enhance the expression of CLDN1 [41, 42], and decrease OCLDN and ZO1 during inflammatory intestinal disease [43]. The results of the present study were in agreement with these previous findings demonstrating that the graded E. maxima infection linearly increased gene expression of JAM2 and CLDN1 but decreased ZO1, OCLDN, and CLDN2. The ZO family is a crucial adapter that connects the other tight junction proteins with cytoskeleton, constructing the intracellular domains of the protein complex [44]. Several binding partners exist for ZO-1, including CLDN, OCLDN, cingulin, and JAM1 [45]. Similarly, ZO-2 co-immunoprecipitates with ZO-1 also presenting binding sections with CLDN and OCLDN [45]. A recent in vitro study revealed that $\mathrm{ZO} 1$ is dominant in the tight junction protein complex in the Madin-Darby bovine kidney cells [46]. It is speculated that ZO-1 may be superior among the ZO family in the tight junction complex of chicken enterocytes, hereby, in the current study, ZO-1 was linearly reduced in response to coccidiosis, whereas ZO-2 was numerically decreased without obtaining significant difference. In addition to the tight junction proteins, the gene expression of MUC2 was linearly reduced following the increased levels of Eimeria inoculation in the current study. A previous study speculated that decreasing mucin production would prevent further intestinal deterioration by limiting Clostridium perfringens infection, as the pathogen tends to utilize mucin for replication [47]. These findings were consistent with another previous report, suggesting that the gene expression of MUC2 is reduced as a consequence of intestinal necrosis [48].

A significant linear reduction in villus height in the jejunum was observed in response to the graded doses of E. maxima in the present study. Because of the reduced absorptive surface area of the brush border in the small intestine, Eimeria-infected chickens possess less space to absorb nutrients from the intestinal lumen. In addition to the short villi in the jejunum, downregulation of nutrient transporters and enzymes at the brush border membrane also contribute to the reduced digestibility of amino acids and energy in coccidiosis $[6,11,12]$. Our previous research also demonstrated a strong correlation $(r=0.76)$ between BW and the ratio of villus height to crypt depth in Eimeria-infected chickens [13], suggesting that intestinal morphology is associated with chicken nutrient digestibility and growth performance.

In conclusion, graded levels of E. maxima infection linearly reduced growth performance and downregulated the expression of nutrient transporters located at the brush border. However, the gene expression of LAT1, CAT1, and GLUT1 was upregulated with increasing challenge doses. Linear regulation of tight junction proteins was observed in 
the current study. This finding was also associated with a linear increase in gastrointestinal permeability in response to the severity of Eimeria infection. Moreover, the greater the number of oocysts challenged to chickens was, the lower the villus height in the jejunum was. Overall, the present study demonstrates the significant impact of graded severity of coccidiosis on several intestine health biomarkers, suggesting that overall reduction of growth performance would be the combined outcome of the reduction of feed intake, gene expression of nutrient transporters, and tight junction proteins by E. maxima infection. The current findings would also help with deciding on an appropriate E. maxima challenge dose in future studies on the evaluation of feed ingredients or feed additives on improving the intestinal health of modern broiler chickens.

\begin{abstract}
Abbreviations
Control: Non-challenged control group; High: High-dose treatment; Medium: Medium-dose treatment; Low: Low-dose treatment; FI: Feed intake; BW: Body weight; dpi: Days post-infection; BWG: Body weight gain; FCR: Feed conversion rate; FITC-d: Fluorescein isothiocyanate dextran; OCLDN: Occludin; ZO1: Zonula occludens 1; ZO2: Zonula occludens 2; JAM2: Junctional adhesion molecule 2; CLDN1: Claudin 1; CLDN2: Claudin 2; MUC2: Mucin 2; GLUT5: Glucose transporter 5; SGLT1: Sodium glucose transporter $1 ; \mathrm{B}^{0} \mathrm{AT}$ : $\mathrm{Na}^{+}$-dependent amino acid transporter; $\mathrm{B}^{0+} \mathrm{AT}$ : $\mathrm{Na}^{+}$-independent amino acid transporter; EAAT: Excitatory amino acid transporter; GLUT1: Glucose transporter 1; GLUT2: Glucose transporter 2; CAT1: Cationic amino acid transporter; LAT2: $\mathrm{Na}^{+}-$ dependent neutral/cationic amino acid transporter; LAT1: L-type amino acid transporter 1.
\end{abstract}

\section{Acknowledgements}

This study was financed in part by a cooperative agreement 58-6040-8-034 from United States Department of Agriculture-Agricultural Research Service. We express our special thanks to Dr. Fuller and all members in Dr. Kim's research group.

\section{Authors' contributions}

P-YT and WKK conceived the research idea and designed the experiment. P-YT and JC carried out the experiments. P-YT and YHT contributed to sample collection and qPCR analyses. P-YT took the lead in writing the manuscript. HL assisted oocysts preparation and interpretation of the result. WKK obtained the grant and supervised the project. HL and WKK reviewed and edited the final version of the manuscript. All authors read and approved the final manuscript.

Funding was provided by USDA-ARS a cooperative agreement 58-6040-8-034.

\section{Declarations}

\section{Competing interests}

The authors declare that they have no competing interests.

\section{Author details}

${ }^{1}$ Department of Poultry Science, University of Georgia, Athens, GA, USA. ${ }^{2}$ U.S. Department of Agriculture-Agricultural Research Center, Beltsville, MD, USA.

Received: 3 March 2021 Accepted: 26 April 2021

Published online: 09 June 2021

\section{References}

1. Blake DP, Knox J, Dehaeck B, Huntington B, Rathinam T, Ravipati V, Ayoade S, Gilbert W, Adebambo AO, Jatau ID, Raman M, Parker D, Rushton J,
Tomley FM (2020) Re-calculating the cost of coccidiosis in chickens. Vet Res 51:115

2. Allen PC (1987) Physiological responses of chicken gut tissue to coccidial infection: comparative effects of Eimeria acervulina and Eimeria mitis on mucosal mass, carotenoid content, and brush border enzyme activity. Poult Sci 66:1306-1315

3. Major JR Jr, Ruff MD (1978) Disaccharidase activity in the intestinal tissue of broilers infected with coccidia. J Parasitol 64:706-711

4. Adams C, Vahl HA, Veldman A (1996) Interaction between nutrition and Eimeria acervulina infection in broiler chickens: development of an experimental infection model. Br J Nutr 75:867-873

5. Su S, Miska KB, Fetterer RH, Jenkins MC, Wong EA (2014) Expression of digestive enzymes and nutrient transporters in Eimeria acervulina-challenged layers and broilers. Poult Sci 93:1217-1226

6. Su S, Miska KB, Fetterer RH, Jenkins MC, Wong EA (2015) Expression of digestive enzymes and nutrient transporters in Eimeria-challenged broilers. Exp Parasitol 150:13-21

7. Paris NE, Wong EA (2013) Expression of digestive enzymes and nutrient transporters in the intestine of Eimeria maxima-infected chickens. Poult Sci 92:1331-1335

8. Turk DE (1972) Protozoan parasitic infections of the chick intestine and protein digestion and absorption. J Nutr 102:1217-1221

9. Sharma VD, Fernando MA, Summers JD (1973) The effect of dietary crude protein level on intestinal and cecal coccidiosis in chicken. Can J Comp Med 37:195-199

10. Sharma VD, Fernando MA (1975) Effect of Eimeria acervulina infection on nutrient retention with special reference to fat malabsorption in chickens. Can J Comp Med 39:146-154

11. Rochell SJ, Parsons CM, Dilger RN (2016) Effects of Eimeria acervulina infection severity on growth performance, apparent ileal amino acid digestibility, and plasma concentrations of amino acids, carotenoids, and alpha1-acid glycoprotein in broilers. Poult Sci 95:1573-1581

12. Teng PY, Yadav S, Dos Santos TS, Fuller AL, Kim WK (2020) 2-Nitro1-propanol improved nutrient digestibility and oocyst shedding but not growth performance of Eimeria-challenged broilers. Poult Sci 99:4314-4322

13. Teng PY, Yadav S, Castro FLS, Tompkins YH, Fuller AL, Kim WK (2020) Graded Eimeria challenge linearly regulated growth performance, dynamic change of gastrointestinal permeability, apparent ileal digestibility, intestinal morphology, and tight junctions of broiler chickens. Poult Sci 99:4203-4216

14. Conway DP, Sasai K, Gaafar SM, Smothers CD (1993) Effects of different levels of oocyst inocula of Eimeria acervulina, E. tenella, and E. maxima on plasma constituents, packed cell volume, lesion scores, and performance in chickens. Avian Dis 37:118-123

15. Sakkas P, Oikeh I, Blake DP, Nolan MJ, Bailey RA, Oxley A, Rychlik I, Lietz G, Kyriazakis I (2018) Does selection for growth rate in broilers affect their resistance and tolerance to Eimeria maxima? Vet Parasitol 258:88-98

16. Cobb Vantress. (2018) COBB broiler management guide. http://cobbsa. co.za/wp-content/uploads/2018/03/BROILER-GUIDE-ENG-2018.pdf. Accessed April 2021

17. Bortoluzzi C, Lumpkins B, Mathis GF, Franca M, King WD, Graugnard DE, Dawson KA, Applegate TJ (2019) Zinc source modulates intestinal inflammation and intestinal integrity of broiler chickens challenged with coccidia and Clostridium perfringens. Poult Sci 98:2211-2219

18. Livak KJ, Schmittgen TD (2001) Analysis of relative gene expression data using real-time quantitative PCR and the 2(-Delta Delta C(T)) method. Methods 25:402-408

19. Adhikari P, Yadav S, Cosby DE, Cox NA, Jendza JA, Kim WK (2020) Research note: effect of organic acid mixture on growth performance and Salmonella Typhimurium colonization in broiler chickens. Poult Sci 99:2645-2649

20. Billard L, Shim MY, Pesti GM (2014) Experimental design and analysis with emphasis on communicating what has been done: (II) calculating interaction contrasts with SAS. Int J Poul Sci 13:88-96

21. Kipper M, Andretta I, Lehnen CR, Lovatto PA, Monteiro SG (2013) Metaanalysis of the performance variation in broilers experimentally challenged by Eimeria spp. Vet Parasitol 196:77-84

22. Byers MS, Howard C, Wang X (2017) Avian and mammalian facilitative glucose transporters. Microarrays 6:7 
23. Fotiadis D, Kanai Y, Palacin M (2013) The SLC3 and SLC7 families of amino acid transporters. Mol Aspects Med 34:139-158

24. Gilbert ER, Li H, Emmerson DA, Webb KE Jr, Wong EA (2007) Developmental regulation of nutrient transporter and enzyme mRNA abundance in the small intestine of broilers. Poult Sci 86:1739-1753

25. Dominguez PA, Pro-Martinez A, Narciso-Gaytán C, Hernández-Cázares A, Sosa-Montes E, Perez-Hernandez P, Caldwell D, Ruiz-Feria CA (2015) Concurrent supplementation of arginine and antioxidant vitamins $E$ and $C$ reduces oxidative stress in broiler chickens after a challenge with Eimeria spp. Can J Anim Sci 95:143-153

26. Castro FLS, Kim WK (2020) Secondary functions of arginine and sulfur amino acids in poultry health: review. Animals 10:2106

27. Perez-Carbajal C, Caldwell D, Farnell M, Stringfellow K, Pohl S, Casco G, Pro-Martinez A, Ruiz-Feria CA (2010) Immune response of broiler chickens fed different levels of arginine and vitamin E to a coccidiosis vaccine and Eimeria challenge. Poult Sci 89:1870-1877

28. Kanai $Y$, Clemencon B, Simonin A, Leuenberger M, Lochner M, Weisstanner M, Hediger MA (2013) The SLC1 high-affinity glutamate and neutral amino acid transporter family. Mol Aspects Med 34:108-120

29. Fetterer $\mathrm{RH}$, Miska KB, Jenkins MC, Wong EA (2014) Expression of nutrient transporters in duodenum, jejunum, and ileum of Eimeria maximainfected broiler chickens. Parasitol Res 113:3891-3894

30. Papaconstantinou HT, Hwang KO, Rajaraman S, Hellmich MR, Townsend CM, Ko TC (1998) Glutamine deprivation induces apoptosis in intestinal epithelial cells. Surgery 124:152-160

31. Papaconstantinou H (2000) Prevention of mucosal atrophy: role of glutamine and caspases in apoptosis in intestinal epithelial cells. J Gastrointest Surg 4:416-423

32. Ryoo HD, Bergmann A (2012) The role of apoptosis-induced proliferation for regeneration and cancer. Cold Spring Harb Perspect Biol 4:a008797

33. Fernando MA, McCraw BM (1973) Mucosal morphology and cellular renewal in the intestine of chickens following a single infection of Eimeria acervulina. J Parasitol 59:493-501

34. Criado-Mesas L, Abdelli N, Noce A, Farre M, Perez JF, Sola-Oriol D, MartinVenegas R, Forouzandeh A, Gonzalez-Sole F, Folch JM (2021) Transversal gene expression panel to evaluate intestinal health in broiler chickens in different challenging conditions. Sci Rep 11:6315

35. Cliffe LJ, Humphreys NE, Lane TE, Potten CS, Booth C, Grencis RK (2005) Accelerated intestinal epithelial cell turnover: a new mechanism of parasite expulsion. Science 308:1463-1465

36. Williams RB (2001) Quantification of the crowding effect during infections with the seven Eimeria species of the domesticated fowl: its importance for experimental designs and the production of oocyst stocks. Int J Parasitol 31:1056-1069

37. Awad WA, Hess C, Hess M (2017) Enteric pathogens and their toxininduced disruption of the intestinal barrier through alteration of tight junctions in chickens. Toxins 9:60

38. Otani T, Nguyen TP, Tokuda S, Sugihara K, Sugawara T, Furuse K, Miura T, Ebnet K, Furuse M (2019) Claudins and JAM-A coordinately regulate tight junction formation and epithelial polarity. J Cell Biol 218:3372-3396
39. Allen PC, Fetterer RH (2002) Recent advances in biology and immunobiology of Eimeria species and in diagnosis and control of infection with these coccidian parasites of poultry. Clin Microbiol Rev 15:58-65

40. Yun $\mathrm{CH}$, Lillehoj HS, Lillehoj EP (2000) Intestinal immune responses to coccidiosis. Dev Comp Immunol 24:303-324

41. Poritz LS, Harris LR 3rd, Kelly AA, Koltun WA (2011) Increase in the tight junction protein claudin-1 in intestinal inflammation. Dig Dis Sci 56:2802-2809

42. Utech M, Ivanov Al, Samarin SN, Bruewer M, Turner JR, Mrsny RJ, Parkos CA, Nusrat A (2005) Mechanism of IFN-gamma-induced endocytosis of tight junction proteins: myosin II-dependent vacuolarization of the apical plasma membrane. Mol Biol Cell 16:5040-5052

43. Scharl M, Paul G, Barrett KE, McCole DF (2009) AMP-activated protein kinase mediates the interferon-gamma-induced decrease in intestinal epithelial barrier function. J Biol Chem 284:27952-27963

44. González-Mariscal L, Betanzos A, Nava P, Jaramillo BE (2003) Tight junction proteins. Prog Biophys Mol Biol 81:1-44

45. Schneeberger EE, Lynch RD (2004) The tight junction: a multifunctional complex. Am J Physiol Cell Physiol 286:1213-1228

46. Beutel O, Maraspini R, Pombo-Garcia K, Martin-Lemaitre C, Honigmann A (2019) Phase separation of zonula occludens proteins drives formation of tight junctions. Cell 179:923-936.e911

47. Forder RE, Nattrass GS, Geier MS, Hughes RJ, Hynd PI (2012) Quantitative analyses of genes associated with mucin synthesis of broiler chickens with induced necrotic enteritis. Poult Sci 91:1335-1341

48. Chen J, Tellez G, Richards JD, Escobar J (2015) Identification of potential biomarkers for gut barrier failure in broiler chickens. Front Vet Sci 2:14

49. Shao Y, Guo Y, Wang Z (2013) beta-1,3/1,6-Glucan alleviated intestinal mucosal barrier impairment of broiler chickens challenged with Salmonella enterica serovar Typhimurium. Poult Sci 92:1764-1773

50. Chen YP, Cheng YF, Li XH, Yang WL, Wen C, Zhuang S, Zhou YM (2017) Effects of threonine supplementation on the growth performance, immunity, oxidative status, intestinal integrity, and barrier function of broilers at the early age. Poult Sci 96:405-413

51. Liu D, Guo S, Guo Y (2012) Xylanase supplementation to a wheat-based diet alleviated the intestinal mucosal barrier impairment of broiler chickens challenged by Clostridium perfringens. Avian Pathol 41:291-298

52. Metzler-Zebeli BU, Magowan E, Hollmann M, Ball MEE, Molnar A, Witter K, Ertl R, Hawken RJ, Lawlor PG, O'Connell NE, Aschenbach J, Zebeli Q (2018) Differences in intestinal size, structure, and function contributing to feed efficiency in broiler chickens reared at geographically distant locations. Poult Sci 97:578-591

53. Paraskeuas V, Mountzouris KC (2019) Broiler gut microbiota and expressions of gut barrier genes affected by cereal type and phytogenic inclusion. Anim Nutr 5:22-31

\section{Publisher's Note}

Springer Nature remains neutral with regard to jurisdictional claims in published maps and institutional affiliations.

\footnotetext{
Ready to submit your research? Choose BMC and benefit from:

- fast, convenient online submission

- thorough peer review by experienced researchers in your field

- rapid publication on acceptance

- support for research data, including large and complex data types

- gold Open Access which fosters wider collaboration and increased citations

- maximum visibility for your research: over $100 \mathrm{M}$ website views per year
}

At BMC, research is always in progress.

Learn more biomedcentral.com/submissions 